$30 \mid 2003$

Les Aventures de Télémaque. Trois siècles

d'enseignement du français. I.

\title{
Les qualités de la langue française d'après les grammairiens contemporains de Fénelon
}

\section{Claude Cortier}

\section{(2) OpenEdition \\ Journals}

Édition électronique

URL : https://journals.openedition.org/dhfles/1561

DOI : $10.4000 /$ dhfles. 1561

ISSN : 2221-4038

Éditeur

Société Internationale pour l'Histoire du Français Langue Étrangère ou Seconde

Édition imprimée

Date de publication : 1 juin 2003

ISSN : 0992-7654

Référence électronique

Claude Cortier, « Les qualités de la langue française d'après les grammairiens contemporains de Fénelon », Documents pour l'histoire du français langue étrangère ou seconde [En ligne], 30 | 2003, mis en ligne le 01 janvier 2012, consulté le 27 mai 2021. URL : http://journals.openedition.org/dhfles/1561 ; DOI : https://doi.org/10.4000/dhfles.1561

Ce document a été généré automatiquement le 27 mai 2021

(c) SIHFLES 


\title{
Les qualités de la langue française d'après les grammairiens contemporains de Fénelon
}

\author{
Claude Cortier
}

1 Durant les XVI ${ }^{e}, \mathrm{XVII}^{\mathrm{e}}$ et XVIII ${ }^{\mathrm{e}}$ siècles, un certain nombre de textes et discours qui font l'éloge des qualités de la langue française se sont constitués parallèlement aux travaux de grammatisation et d'outillage de la langue vulgaire qu'était alors le français pour lui permettre d'accéder au statut de langue classique, à l'instar du latin, puis de langue digne d'une grande puissance européenne : travaux de traduction des textes anciens entrepris dès le $\mathrm{XV}^{\mathrm{e}}$ siècle : la traduction conduit à introduire des mots nouveaux, de nouvelles tournures de pensée et à enrichir la langue ; travaux de grammatisation initiés au XVI ${ }^{e}$ siècle et poursuivis au XVII ${ }^{e}$ siècle ; travaux littéraires de divers genres servant de corps de référence et d'analyse.

2 Ces discours, parfois à teneur de " propagande », ont permis, au XVI e siècle surtout, de fournir à ceux qui étaient disposés à s'émanciper du latin de nouveaux arguments et de rallier des partisans : on y défend la richesse de la langue, puis la volonté de normalisation qui lui permet d'acquérir continuité et stabilité dans le temps (Longeon 1989 : 17).

$3 \mathrm{Au} \mathrm{XVII}{ }^{e}$ siècle, les discours sur les qualités sont, nous semble-t-il, la contrepartie des travaux quelque peu « besogneux » des salons et des académies, « des petits cercles de beaux esprits rassemblés sous l'autorité de l'Académie, qui se dépensent à organiser une langue pure » (Chevalier 1997 : 3398), nette et claire. «Tout un peuple, restreint [...] devient sensible à ce qu'il écrit, "à un usage réfléchi et conscient" et se livre à l'exploration des types généraux humains en fouillant la langue " (ibid., citant Brunot, H.L.F., III, V). À la fin du siècle, l'exigence de pureté, de netteté et de concision associée à l'ordre de la raison se confondra dans la clarté, pièce maîtresse du génie ${ }^{1}$ de la langue (Swiggers 1987). Le XVIII e siècle sera celui du rayonnement de « la langue française cultivée » et de son universalisation. 
Les linguistes à partir de la fin du XIX ${ }^{e}$ siècle, notamment Ferdinand Brunot (1896), ont contesté la validité de ces discours et plus particulièrement le mythe de la clarté et de l'ordre naturel, ils ont également condamné les manifestations corollaires de chauvinisme ou « xénophobie linguistique ».

Il nous semble cependant, à l'instar d'un groupe de linguistes réunis par Jean-Michel Éloy (1995), que l'historien et le sociolinguiste peuvent les prendre en compte pour mieux cerner les phénomènes et les représentations qui participent à la connaissance d'une époque. Leur mise en perspective avec les écrits de Fénelon sur l'éloquence et la poésie permettra de compléter cette approche et de mieux comprendre la réception et la diffusion d'une œuvre comme le Télémaque de Fénelon dont le style se veut modélisant pour l'éducation².

\section{Grammaticalisation et normalisation}

\section{Création de l'Académie française}

Comme chacun sait, c'est au XVII ${ }^{\mathrm{e}}$ siècle essentiellement que s'est développé le processus de formation de la langue classique, grâce aux travaux de Malherbe puis des nombreux " grammairiens ", " observeurs » et "remarqueurs " qui ont permis au français d'être passé au crible, pesé, puis fixé par un corps officiellement revêtu des pouvoirs nécessaires et sans analogue dans l'Antiquité : la création de l'Académie française en 1635 par Richelieu fut en effet le premier acte décisif de ce siècle en matière linguistique. Les premiers travaux des académiciens ont porté sur l'examen des textes des écrivains disparus, tel Malherbe, afin d'en tirer des règles et des exemples sur la langue. Puis, on s'intéressa au Cid de Corneille avant d'entreprendre, sous la direction de Vaugelas, la préparation du Dictionnaire qui allait occuper l'Académie jusqu'à la fin du siècle (1694).

7 Fénelon entra à l'Académie en 1693, où il remplaçait Pellisson (cf. Discours de réception). Il proposa en 1714, suite à une demande de l'institution, des Réflexions sur la Grammaire, la Rhétorique, la Poétique et l'Histoire, communément appelées Lettre à l'Académie. L'étendue et l'importance de l'ouvrage, la situation éminente de l'auteur déterminèrent les académiciens à le faire imprimer; il parut en 1716. Ces Lettres correspondent à des travaux que Fénelon suggère à l'Académie de mettre en oeuvre dans l'intérêt de la langue française : " Projet d'achever le Dictionnaire, Projet de grammaire, Projet d'enrichir la langue, Projet de rhétorique, Projet de poétique, Projet d'un traité sur la tragédie, Projet d'un traité sur l'histoire ». Fénelon y développe également un point de vue critique sur l'évolution imposée à la langue par le courant normatif le plus puriste (Vaugelas, Bouhours) et formule un certain nombre de jugements et propositions sur les qualités de la langue à développer dans les différents genres et styles.

\section{Le règne et l'autorité de Vaugelas}

8 Parmi les académiciens, figurent un certain nombre de " grammairiens $»^{3}$ dont le plus connu fut sans conteste Vaugelas. Les principes qui guidèrent ses travaux sont développés dans la Préface des Remarques sur la langue française parues en 1647, Remarques qui sont à la fois toute son œuvre et la référence des puristes. Le titre même de l'ouvrage mérite éclaircissement. Les Remarques ne sont pas des lois, ni des décisions, 
Vaugelas prétend passer pour un simple témoin qui consigne ce qu'il a vu et entendu et non un juge. " Il n'y a », dit-il, « qu'un maistre des langues, qui en est le Roy et le tyran, c'est l'Usage " (Préface : II). La Cour (hommes et femmes, personnes de la ville où le prince réside) est en quelque sorte le "magasin de la langue ", c'est elle qui contribue pour la plus grande part à former l'usage, le langage des bons auteurs « en est comme une vérification ". On peut y joindre encore "l'avis des gens savants en la langue " (ibid.). Le « bon usage » est le résultat d'un contrat relevant de la liberté des particuliers en tant qu'ils ont contrat ensemble, en tant qu'ils forment un "public ». Ainsi l'usage est à la fois « une puissance souveraine témoignant d'une volonté coutumière et comme une source d'obligations pour tous en général et en particulier » (Merlin-Kajman 2003 : 145). La langue des puristes est le résultat d'un tel contrat mais elle en est aussi le témoin (ibid. : 146) et le gage. Ainsi peut se constituer une société des praticiens du « bon usage ", une société civile qui par correspondances et mimétisme pourra s'étendre dans toute l'Europe.

Ce règne de l'usage sera néanmoins contesté en France dans la seconde moitié du siècle avec la publication d'abord de la Grammaire générale et raisonnée (1660) d'Arnault et Lancelot puis du Discours sur l'usage dans la langue françoise de Grimarest. Alors que les grammairiens du bon usage ont une démarche empirique - c'est à partir d'exemples qu'ils distinguent le bon et le mauvais usage et établissent une règle prescriptive - la Grammaire générale recherche les principes généraux de nature logique qui procèdent $\mathrm{du}$ fonctionnement de l'esprit humain : l'expression doit être en conformité avec les opérations de l'esprit. Quant à l'ouvrage de Grimarest, c'est la négation de tous les principes proclamés par Vaugelas, une révolte contre les courtisans « qui ne possèdent que le quart de la langue, à la fantaisie desquels il faut substituer les rigueurs de la logique »(Brunot, $H L F$, IV : 57).

\section{L'œuvre de Bouhours}

Comme on peut le constater dans l'éventail de ses publications de 1671 à $1689^{4}$, l'œuvre de Bouhours est sans doute l'une des plus complètes de ce siècle dans la description des rapports entre langue, style et pensée. Ce sont Les Entretiens d'Ariste et d'Eugène (1671), son premier ouvrage, qui l'ont rendu célèbre ${ }^{5}$, notamment parce qu'il y poursuit, dans le domaine de la grammaire et du bon usage, ses attaques à Port-Royal, provoquant les réactions de son adversaire, Gilles Ménage, favorable aux jansénistes. Tout en reconnaissant que ces Messieurs " ont beaucoup contribué à la perfection de notre langue », Bouhours s'attaque aux « discours vastes » et aux " périodes démesurées » qui caractérisent les écrits des théologiens. Ceci explique que les ouvrages de Bouhours aient suscité de nombreuses réponses et polémiques, qui ont contribué à sa renommée, en France et à l'étranger. Les contemporains s'accordent à souligner les emprunts de Bouhours notamment à Pasquier et à Le Laboureur. Ce sont pourtant les Entretiens que la postérité a retenus : ils sont connus pour être un modèle de défense et d'illustration de la langue française au $\mathrm{XVII}^{e}$, tout en comportant un certain nombre de remarques ou raisonnements désobligeants ${ }^{6}$ à l'égard des langues italienne et espagnole notamment, que Bouhours, comme Ménage d'ailleurs, connaissait bien. 


\section{Des travaux du grammairien aux qualités de la langue}

11 La publication suivante de Bouhours, Doutes sur la langue françoise proposés à Messieurs de l'Académie par un gentilhomme de province (1674), nous éclaire sur le labeur du " remarqueur ", qu'il nous semble utile de mettre en regard des discours sur les qualités, dont seul il peut fonder la légitimité. L'ouvrage est divisé en cinq parties : Doutes sur les mots, Doutes sur les phrases, Doutes sur la construction, Doutes sur la netteté, Doutes sur ce qui regarde l'exactitude du style.

12 Dans chacune des parties, la méthode est à peu près identique, il s'agit de relever des emplois dans des textes des bons auteurs, de vérifier dans les observations ou remarques publiées ce qu'en disent les contemporains, de rechercher dans l'usage des formes attestées. Par exemple, les mots commençant par in-donnent lieu au récit de longs échanges avec d'autres « remarqueurs », courtisans ou « honnestes gens » dont certains propos sont cités dans le détail lorsqu'ils permettent d'attester un usage (Doutes sur les mots : 19-30).

13 Pour chaque cas, Bouhours propose un point de vue qu'il soumet finalement à l'appréciation de l'Académie.

14 D'après les remarques que nous avons pu collecter au fil du texte, les qualités de la langue qui sont mises en évidence dans les Doutes sont :

15 - l'appartenance au " génie de la langue »;

16 - concernant la construction de la phrase, la régularité ;

17 - concernant la construction de la phrase, la netteté ;

18 - concernant la construction de la phrase, l'arrangement et l'ordre des mots.

\section{La clarté}

$19 \mathrm{Au}$ XVII ${ }^{\mathrm{e}}$ siècle, la clarté, c'est d'abord l'ordre et la place des mots, l'arrangement. Cet ordre jusque là n'était pas fixé rigoureusement de telle sorte que les écrivains usaient d'une assez grande liberté, l'habitude des langues à flexion contribuant à cette tradition. Les irrégularités de construction, les ambiguïtés (problèmes d'anaphores), les constructions « louches » ou " obscures » et le non respect de l'ordre naturel nuisent à la clarté de la langue. Le travail de la plupart des grammairiens va principalement porter sur deux points : renoncer à séparer les divers éléments d'une même forme et rapprocher l'un de l'autre les termes de la phrase qui sont en rapport logique (le sujet du verbe, le participe passé de l'auxiliaire, le verbe du relatif régime complément, la préposition de son complément, etc.).

20 La recherche de la clarté devient l'objet de scrupules infinis : « Si le lecteur relit deux fois une période, ce doit estre pour l'admirer et pour le plaisir qu'il a de repeter les belles choses, mais non pas pour chercher ce que l'Autheur a voulu dire. » $(1674: 183)$. La longueur des périodes doit également être mesurée avec soin, si on veut éviter l'embarras, une période trop longue fatigue car « nostre humeur prompte ne s'accorde pas avec cette ennuyeuse attente » (ibid.).

21 Le principe majeur qui guide la recherche de clarté de la langue française est celui de l'ordre naturel des mots (sujet-verbe-objet). Cet ordre est dit «naturel » car il correspond à « la manière dont les pensées naissent dans l'esprit » (1671:56). L'ordre 
naturel est présenté comme une forme de la naïveté, correspondant à une expression spontanée : « Il n'y a que la langue française qui suive la nature pas à pas [...] ; et elle n'a qu'à la suivre fidèlement pour trouver le nombre et l'harmonie que les autres langues ne rencontrent que dans le renversement de l'ordre naturel » (ibid.).

Mais cette nature est aussi raison. La clarté de l'expression est indissociable du bon sens ou clarté du raisonnement " la raison n'étant pas moins essentielle au style qu'à l'homme » $(1674: 221)$; elle est ennemie « du galimatias et de tout ce qui choque le bon sens ». Il faut rechercher " ce que demande Quintilien et qui consiste à rendre le langage plausible aux doctes, clair aux ignorants "(ibid. : 228). Ce souci de la compréhension par tous est une attestation de la vocation didactique du français et de sa prétention à l'universalité, non seulement géographique, mais aussi sociale.

\section{Une richesse façonnée}

Selon Bouhours (1671 : 104-107), les travaux du XVII ${ }^{e}$ siècle doivent mettre de l'ordre dans l'héritage des siècles précédents durant lesquels la langue s'est considérablement enrichie, certes, mais dans le foisonnement et l'exubérance. Il s'agit donc d'inverser le mouvement et de construire une richesse façonnée : c'est-à-dire travailler le sens des mots, le délimiter précisément, de façon à ce que chaque terme puisse être employé proprement. Le nombre de mots importe moins que la richesse, la finesse et complexité de leur sens : la vraie richesse est ainsi celle de la qualité.

Le façonnement de la langue implique un travail d'épuration et de polissage, il faut " polir » et supprimer les « ordures », retrancher « tout ce que la langue peut contenir de barbare " (1671: 77). Le terme " polir » est révélateur du travail des grammairiens (métaphore des arts et métiers) mais aussi de ceux qui s'efforcent de respecter le bon usage.

De la même façon que l'on polit, l'on va retrancher : il faut travailler durement et supprimer tout ce " qui dépasse ", tout excès, toute aspérité, toute gêne, tout ce qui peut diviser le sens (une construction louche fait regarder dans deux directions) et donc « diviser les locuteurs » (Merlin-Kajman 2003 : 125).

Dans le domaine du style, on va supprimer les excès et exagérations qui sont représentées par les figures : la métaphore, l'hyperbole, les superlatifs, tout ce qui témoigne de l'embellissement forcé, de l'artifice, des fausses beautés comme le sont les fards et les masques. Si l'on parvient à éliminer tout excès, toute figure, tout néologisme, toute affectation, toute composition, en refusant les termes trop recherchés, les phrases trop élégantes, les périodes compassées, on pense pouvoir conduire la langue à sa perfection.

L'art de la langue doit procéder comme la joaillerie ou l'orfèvrerie : une langue est une œuvre, qui s'élabore comme un objet ou mieux une oeuvre d'art, peinture ou sculpture, grâce à un travail, tout en finesse, la finesse étant ce qui présente un caractère de perfection ou une extrême acuité.

28 Cependant, la comparaison avec le latin et les langues romanes, les excès et les défauts des autres langues permettent de dégager par contraste une forme de simplicité naturelle. La langue française permet d'« exprimer les choses précisément comme elles sont ", de peindre d'après nature, car " Tout ce qui sent l'étude, tout ce qui a l'air de contrainte choque » $(1671: 52)$. 
29 La langue polie est celle d'une société polie, où règnent la politesse et la bienséance. Ces qualités s'acquièrent par le " commerce des honnestes gens et la lecture des bons livres» (ibid. : 124) : la lecture apporte la netteté et la justesse du style, la conversation apprend à parler noblement et naturellement. Elles se manifestent dans l'exercice et l'art de la parole, chez ceux qui ont " pour ordinaire cet air aisé et naturel qui est si fort à la mode " (ibid. : 125. Nous soulignons) et qui réunissent, par un génie inné de la langue, des qualités difficiles à allier : " une grande facilité et une grande exactitude ; des paroles harmonieuses, mais pleines de sens ; de la brièveté et de la clarté ; une expression fort simple et en même temps fort noble, une extrême pureté et une naïveté admirable » (ibid. : 145).

Ces propos montrent d'évidence l'utilisation, qui deviendra de plus en plus systématique, d'adjectifs désignant des qualités humaines pour décrire des qualités de la langue. Une correspondance s'instaure entre les qualités de l'homme et celles de la langue dans laquelle il s'exprime, c'est ainsi que l'on définit « l'honneste homme », on comprend alors comment l'éducation à l'élocution, à la politesse, par l'acquisition des " tours » et des « airs » se présente comme une pièce maîtresse de l'éducation morale. " L'air » est un art de dire et d'être socialement, qui donne à voir et entendre la pureté et la clarté de la langue classique.

\section{Les points de vue de Fénelon}

31 Les arguments de Bouhours en faveur d'une richesse façonnée de la langue, qui exclut les archaïsmes et les néologismes et se méfie des sens figurés ne feront pas l'unanimité. De nombreuses voix, dont celle de Fénelon dans la Lettre à l'Académie (désormais L.A.) s'élèveront contre cette norme sélective, ce perfectionnisme réducteur :

Notre langue manque d'un grand nombre de mots et de phrases : il me semble même qu'on l'a gênée et appauvrie depuis environ cent ans en voulant la purifier. [...]. On a retranché, si je ne me trompe, plus de mots qu'on en a introduit. D'ailleurs je voudrois n'en perdre aucun, et en acquérir de nouveaux. (L.A. Projet d'enrichir la langue : 473).

32 Fénelon propose d'assouplir les principes des puristes en autorisant les emprunts, notamment au latin, en retenant « tout terme qui nous manque et qui a un son doux, sans danger d'équivoque "(ibid.). Il souligne qu'il n'existe pas ou peu de vrais synonymes et que la plupart des termes ont besoin d'être qualifiés par une épithète pour être suffisamment précis. Il formule ensuite un principe de style dont Françoise Berlan (1990) ${ }^{7}$ discute l'application au premier livre de Télémaque :

Je voudrois même plusieurs synonymes pour un seul objet : c'est le moyen d'éviter toute équivoque, de varier les phrases et de faciliter l'harmonie, en choisissant celui qui sonneroit le mieux avec le reste du discours (ibid.).

33 Si Fénelon critique le purisme en matière de vocabulaire, il semble s'accorder avec Bouhours pour rapprocher « nature » et « naturel » sur la voie de la simplicité :

On a même senti de nos jours que le style fleuri, quelque doux et agréable qu'il soit ne peut jamais s'élever au-dessus du genre médiocre, et que le vrai genre sublime, dédaignant tous les ornements empruntés, ne se trouve que dans le simple. (Discours de réception à l'Académie : 535).

Il ne s'agit pas de supprimer toutes les figures, mais seulement celles qui n'apportent que pur ornement ou affectation. On conservera en revanche les ornements " qui servent à démêler ce qui est obscur ", à " peindre vivement ce qu'on veut mettre 
devant les yeux ", à " prouver une vérité par divers tours sensibles ", à " remuer les passions, qui sont les seuls ressorts capables d'intéresser et de persuader l'auditeur ; car la passion est l'âme de la parole " (ibid.). L'utilisation des images ici préconisée correspond aux intentions du pédagogue et du prédicateur. Il s'agit de toucher le lecteur, de lui rendre les choses sensibles pour l'amener à sentir, à se représenter les faits, à comprendre la manière dont ils sont arrivés $(1718: 35)$.

De même qu'il critique l'affectation, Fénelon s'élève à plusieurs reprises contre les abus du " bel esprit ", les démonstrations d'ingéniosité, susceptibles de pencher vers la frivolité. Dans les Dialogues sur l'éloquence, il peut apparaître comme une sorte d'antiBouhours (1718: 17, 54 ; Berlan 1990 : 234). Il reprendra cette critique dans le discours de réception à l'Académie :

Le goût exquis craint le trop en tout sans en excepter l'esprit même. L'esprit lasse beaucoup, dès qu'on l'affecte et le prodigue. C'est en avoir de reste que d'en savoir retrancher pour s'accommoder à celui de la multitude, et pour lui aplanir le chemin. Les poëtes qui ont le plus d'essor, de génie, d'étendue de pensées et de fécondité sont ceux qui doivent le plus craindre cet écueil de l'excès d'esprit. (L.A. Projet de poésie : 500 )

Un auteur qui a trop d'esprit fatigue le lecteur, car il ne le laisse pas respirer, la lecture devient une étude. Aux modernes « ingénieux », Fénelon préfère les anciens qui n’ont que « la simple nature ».

Je demande un poëte aimable, proportionné au commun des hommes, qui fasse tout pour eux et rien pour lui. Je veux un sublime si familier, si doux, si simple, que chacun soit d'abord tenté de croire qu'il l'aurait trouvé sans peine, quoique peu d'hommes soient capables de le trouver. Je veux un homme qui me fasse oublier qu'il est auteur et qui se mette de plain-pied avec moi. (ibid.)

La véritable éloquence se modère et se proportionne aux sujets qu'elle traite :

Il faut être grand dans les grandes choses ; il faut être simple, sans être bas dans les petites, il faut tantôt de la naïveté et de l'exactitude, tantôt de la sublimité et de la véhémence. (Dialogues: $\mathrm{I}, 7$ )

Le goût de Fénelon pour l'authenticité du sentiment révèle le prédicateur et l'éducateur, la simplicité du style doit rejoindre celle de l'homme : "Ô qu'il y a de grandeur à se rabaisser ainsi pour se proportionner à tout ce qu'on peint » (ibid. : 501).

C'est pour les mêmes raisons que Fénelon revendique comme Bouhours l'art de peindre d'après nature : «On a enfin compris, messieurs, qu'il faut écrire comme les Raphaëls, les Carraches et les Poussins ont peint, non pour chercher de merveilleux caprices et pour faire admirer leur imagination en se jouant du pinceau, mais pour peindre d'après nature " (Discours de réception : 535). Mais il ne s'agit pas de confondre simplicité et sécheresse, d'être « sec et vague dans l'expression » comme dans beaucoup d'œuvres contemporaines où " tout se passe en exclamations sans preuve et sans peinture » (Dialogues : II, 6). Pour cela on peut imiter les Grecs qui se servaient peu de termes généraux et " disaient beaucoup de faits » sans pratiquer la " fausse politesse » de « certains provinciaux qui se piquent de bel esprit » qui consiste à ne point dire ce qui est utile parce qu'on ne peut l'embellir :

Tout entre dans les sujets que l'éloquence doit traiter. La poésie même qui est le genre le plus sublime ne réussit qu'en peignant les choses avec toutes leurs circonstances. [...] En faisant un détail, il ne faut rien présenter à l'esprit de l'auditeur qui ne mérite son attention, et qui ne contribue à l'idée qu'on veut lui donner. Ainsi il faut être judicieux pour le choix des circonstances ; mais il ne faut point craindre de dire tout ce qui sert (ibid. : 57). 
les Dialogues, comme dans la Lettre Fénelon s'attaque également avec véhémence au principe de l'ordre naturel qui, conjugué à l'obligation de rimes riches, rend difficile la versification en langue française :

On s'est mis à pure perte dans une espèce de torture pour faire un ouvrage. Nous serions tentés de croire qu'on cherche le difficile plutôt que le beau. [...] Au contraire, les Anciens facilitaient, par des inversions fréquentes, les belles cadences, la variété, et les expressions passionnées. Les inversions se tournaient en grande figure, et tenaient l'esprit suspendu dans l'attente du merveilleux. (L.A. Poétique : 498).

41 Certes, il ne faudrait sans doute pas introduire tout à coup ces inversions en trop grand nombre comme Ronsard a pu le faire, mais «l'excès choquant de Ronsard nous a un peu jetés dans l'extrémité opposée " et Fénelon de décrire dans un style parfaitement cadencée, la règle de l'ordre naturel défendue par Bouhours :

On a appauvri, desséché et gêné notre langue. Elle n'ose jamais procéder que suivant la méthode la plus scrupuleuse et la plus uniforme de la grammaire : on voit toujours un nominatif substantif, qui mène son adjectif comme par la main, son verbe ne manque pas de marcher derrière, suivi d'un adverbe qui ne souffre rien entre les deux ; et le régime appelle aussitôt un accusatif, qui ne peut jamais se déplacer. C'est ce qui exclut toute suspension de l'esprit, toute attention, toute surprise, toute variété et souvent toute magnifique cadence (L.A. : ibid.).

Ainsi, en dépit de quelques similitudes avec les maximes de Bouhours "peindre en suivant la nature ", " Pour plaire, il ne faut point chercher à plaire » (1671:53), la description du beau style chez l'un ou du beau langage chez l'autre montre deux conceptions souvent opposées. L'idéal de Bouhours est décrit par la métaphore de l'eau pure :

Le beau langage ressemble à une eau pure et nette qui n'a point de goût [...] la langue française hait encore tous les ornements excessifs : elle voudrait presque que toutes ses paroles fussent nues, pour s'exprimer plus simplement; elle ne se pare qu'autant que la nécessité et la bienséance le demande (ibid.).

Si Fénelon aspire également à un art dépouillé, proche de l'idéal antique, s'« il y a une bienséance à garder pour les paroles comme pour les habits ", si « l'éloquence est un art sérieux qui ne supporte pas l'affectation du genre fleuri », l'orateur doit adapter son discours à l'auditoire :

On ne doit user des expressions qui plaisent qu'à cause qu'il y a peu d'hommes assez raisonnables pour goûter une vérité qui est sèche et nue dans le discours (L.A., Rhétorique : 479).

L'orateur moderne doit se montrer digne d'être écouté, c'est un homme "à l'âme forte, mais dont les moindres discours auront des traits originaux que les déclamateurs fleuris ne pourront jamais imiter ». Il n'orne son discours que " de vérités lumineuses, que de sentiments nobles, que d'expressions fortes et proportionnées à ce qu'il tâche d'inspirer » (ibid. : 487).

Les règles de Saint Augustin qu'il propose de suivre sont celles d'une éloquence sérieuse et efficace :

Il faut parler d'une façon abaissée et familière pour instruire ; d'une façon douce, gracieuse et insinuante pour faire aimer la vérité, d'une façon grande et véhémente, quand on a besoin d'entraîner les hommes et de les arracher à leurs passions. (ibid. : 479)

On sait que le style du Télémaque est aux antipodes de l'idéal de Bouhours, de « cette eau pure, nette et sans goût ", sans pour autant sacrifier à la rigueur qui se manifeste dans 
la simplicité et l'architecture du texte : rythmes binaires, parallélismes, symétrie (Berlan 1990 : 241). La composition de la phrase suit un cours régulier, elle doit sa fluidité à la parataxe, coordination et juxtaposition, à une " ordonnance claire des éléments dans des séquences courtes "(ibid. : 239). Les expressions imagées, les épithètes plus souvent appréciatives que descriptives favorisent cette intrusion de l'homme dans le discours, cette "douce insinuation " propre à se faire entendre et aimer (L.A : 479) qui laisse parfois place à une hausse de ton, à une vigueur, voire une véhémence nécessaire pour entraîner à l'action. Cependant ce mouvement, cette force ne doivent avoir leur origine que dans la nature, face à laquelle l'homme doit rester humble. Son art doit se confondre avec la nature même, car « l'art se trahit en se montrant» (ibid. : 490).

À la volonté d'éducation par la langue des grammairiens, conjuguant art de la politesse et rigueur de l'expression, Fénelon, prédicateur, éducateur et pédagogue ajoute le pouvoir de la poésie, "plus sérieuse et utile qu'on le croit» (ibid.), le pouvoir des images peintes d'après nature pour développer une morale sensible et aimable, accessible à tous. Le succès de Télémaque a pu en témoigner.

\section{BIBLIOGRAPHIE}

\section{Bibliographie}

BERLAN, Françoise (1986), « Du lexique au style : Fénelon », XVII siècle 15, 231-244.

-- (1990), « Fénelon, traducteur et styliste, réécriture du chant V de l'Odyssée », Littératures classiques 13, 19-52.

BRUNOT, Ferdinand (1896), « Discours prononcé lors de la cérémonie de l'ouverture des cours de l'Alliance française », Bulletin de l'Alliance française 61, août-septembre.

-- (1909 et 1911), Histoire de la langue française des origines à nos jours, III : La formation de la langue classique 1600-1660. $1^{\text {ère }}$ partie ; IV : La langue classique 1660-1715, Paris, A. Colin.

BOUHOURS, Dominique (1671), Entretiens d'Ariste et d'Eugène, Paris, Mabre-Cramoisy. Édition consultée : Paris, Éditions Bossard, introduction et notes de René Radouant, 1920.

------(1674), Doutes sur la langue française proposés à Messieurs de l'Académie Française par un gentilhomme de province, Paris, Mabre-Gramoisy. Édition consultée : London and Tonbridge, Lewis Reprints Limited, 1971.

------(1675), Remarques nouvelles sur la langue française, Paris, Mabre-Gramoisy.

------(1687), La manière de bien penser dans les ouvrages d'esprit. Dialogues, Nouvelle édition, Paris, Brunet, 1715. Édition consultée, Brighton, Sussex reprints, University of Sussex Libretti, 1971.

CHEVALIER, Jean-Claude (1997), « L'Histoire de la langue française », in Nora (dir.), 3385-3419. 
CHÉREL, A. (1994), « L'idée du naturel et le sentiment de la nature chez Fénelon », in LANAVÈRE dir., 77-92.

ÉLOY, Jean-Michel dir. (1995), La qualité de la langue ? Le cas du français, Paris, Champion.

FÉNELON (1699), Discours prononcé dans l'Académie française le mardi 31 mars 1693 à la réception de Monsieur l'abbé de Fénelon, précepteur du Duc de Bourgogne et de Monseigneur le Duc d'Anjou, in CEuvres complètes (1983), I. Édition établie par Jacques Le Brun, Paris, Gallimard, 533-539.

------(1718), Dialogue sur l'éloquence en général et sur celle de la chaire en particulier, in Cuvres complètes (1983), cité, 3-87 (1ère édition par Ramsay en 1718, rédaction probable entre 1677 et 1681, cf. Le Brun : 1233).

------(1716), Réflexions sur la Grammaire, la Rhétorique, la Poétique et l'Histoire, ou Lettre à l'Académie, Paris, Coignard (texte adressé à l'Académie et lu en séance du 26 mai 1714). Éditions consultées : Firmin-Didot et Cie, Paris, 1875 ; édition critique par Ernesta Caldarini, Genève, Droz, 1970.

FUMAROLI, Marc (1997), « Le génie de la langue française », in Nora (dir.), 4623-4685.

LANAVÈRE, Alain dir. (1994), Je ne sais quoi de pur et sublime... Télémaque, Orléans, Paradigme.

LEROY-TURCAN, I., WOOLDRIDGE, T.R. dir. (1998), Gilles Ménage (1613-1692), grammairien et lexicographe. Le rayonnement de son oeuvre linguistique, Actes du colloque international tenu à l'occasion du tricentenaire du Dictionnaire étymologique ou Origines de la langue françoise (1694), Université Jean Moulin Lyon III, 17-19 mars 1994. Édition électronique, Toronto, SIEHLDA, 1998 et EDICTA, 2000.

LONGEON, C. (1989), Premiers combats pour la langue française, Paris, Le livre de poche.

MC KENNA, A. $(1998,2000)$, « Ménage et Bouhours », in LEROY-TURCAN / WOOLDRIDGE dir., 95-120.

MERLIN-KAJMAN, H. (2003), La langue est-elle fasciste?, Paris, Seuil.

PELLAT, J.C. (1998, 2000), « Ménage, un continuateur de Vaugelas influencé par Port-Royal ? », in LEROY-TURCAN, I. et WOOLDRIDGE, T.R. dir., 121-141.

NORA, P. dir. (1997), Les lieux de mémoire, 3, Paris, Quarto Gallimard.

SWIGGERS, Pierre (1987), « À l'ombre de la clarté française », Langue française 75, 5-21.

VAUGELAS, G.F. (1647), Remarques sur la langue française ; édition consultée : Fac simile de l'édition originale établie par Jeanne Streicher, Genève, Slatkine Reprints.

WILMET, Marc dir. (1987), « La clarté française », Langue française 75, 5-10.

\section{NOTES}

1. Le génie d'une langue désigne les qualités qui lui sont propres et qui permettent de la distinguer des autres langues (Fumaroli 1997).

2. Cf., dans cette même publication, Anne-Marie Perrin-Naffakh, « Télémaque, un style exemplaire?».

3. C'est par commodité que nous les désignerons désormais sous cette appellation, car la plupart d'entre eux n'ont pas rédigé de grammaire, ils ont analysé et codifié les usages.

4. Entretiens d'Ariste et d'Eugène, 1671. Doutes sur la langue française proposés à Messieurs de l'Académie Française par un gentilhomme de province, Paris, 1674. Remarques nouvelles sur la 
langue française, 1692. La manière de bien penser dans les ouvrages d'esprit. Dialogues, 1687. Pensées ingénieuses des Anciens et des Modernes, 1689.

5. Ce texte vient de faire l'objet d'une réédition aux éditions Champion (2003), où il est présenté comme « l'un des textes les plus représentatifs et à bien des titres fondateurs du classicisme des années 1660-1670, demeuré jusqu'ici en marge du renouveau des études dix-septiémistes ».

6. Formes de "xénophobie linguistique " évoquées dans notre introduction qui ne peuvent se justifier que dans la mesure où ces grammairiens ont cherché à différencier la langue française des autres langues latines, candidates antérieurement à la précellence européenne, à la spécifier, à déterminer son " génie ".

7. Son étude précise et statistique du vocabulaire et du style permet de montrer que si la synonymie en tant que telle joue un faible rôle, le vocabulaire s'organise en vastes ensembles, en réseaux de filiation et de proximité sémantique dans lesquels les adjectifs épithètes, souvent par paires, à la fois descriptifs et évaluatifs servent la peinture du décor et la morale de l'histoire.

\section{RÉSUMÉS}

Les discours sur les qualités de la langue française accompagnent l'œuvre de grammatisation et de normalisation de la langue en même temps qu'ils participent au mouvement d'émancipation et à " l'universalisation » du français. L'étude conjointe des travaux des grammairiens et remarqueurs du XVII ${ }^{\mathrm{e}}$ siècle et des discours d'éloge montre les liens qui se tissent entre les qualités de la langue et celles de l'homme. À cette volonté d'éducation par l'art de la politesse et la rigueur de l'expression, Fénelon ajoute le pouvoir de la poésie, des images peintes d'après nature pour développer une morale sensible et aimable.

The discurses about French langage properties occur with the grammatization and langage normalization; in the same time they take part in the emancipation and universalization of the French langage. The study of grammarians works and langage praising discurses shows the links between langage properties and human qualities. Fénelon oversteps this current education (politness and style); he adds the poetry power and the painted from nature to develop a sensitive and honorable morality.

\section{INDEX}

Keywords : education, politness, style, normalization, grammatization, grammairians, XVIIth century

Mots-clés : éducation, politesse, style, normalisation, grammatisation, remarqueurs, XVII siècle

\section{AUTEUR}

CLAUDE CORTIER

INRP, UMR 5191 (CNRS Lyon 2, ENS-LESH) - Lyon 